\title{
IMPACTO DE LA POLÍTICA PÚBLICA \\ ETNOEDUCATIVA COLOMBIANA EN EL \\ CURRÍCULO DE LAS INSTITUCIONES EDUCATIVAS \\ AFRODESCENDIENTES: CASO INSTITUCIÓN \\ EDUCATIVA ANTONIA SANTOS, CARTAGENA DE \\ INDIAS, COLOMBIA
}

\author{
Oscar Javier Ospino Guerrero ${ }^{1}$ \\ Osiris Castilla Tijera² \\ Alejandrina Lago De Zota ${ }^{3}$
}

\begin{abstract}
Resumen
En los últimos años la legislación colombiana le ha apostado a una educación inclusiva, étnica y multicultural. Estas políticas son el resultado de muchos años de lucha de algunas minorías como los afrodescendientes que históricamente han sido discriminadas, excluidas e invisibilizadas. Este trabajo pretende evidenciar la política pública etnoeducativa colombiana y su influencia en el currículo de las instituciones de educación con una marcada identidad afro, tomando como referencia la Institución Educativa Antonia Santos de Cartagena, Colombia. Para lograr este
\end{abstract}

\footnotetext{
1 Docente Institución Etnoeducativa e Inclusiva Antonia Santos. Magíster: E-Learning. Universidad Autónoma de Bucaramanga (UNAB). - Universidad Oberta de Cataluña (UOC). Semillero grupo de Investigación RUECA. Correo: oscarsavier@hotmail.com

2 Docente Etnoeducativa, Licenciada en Preescolar y promoción social de la familia. Interprete en lengua de señas colombiana Institución educativa Antonia Santos. Semillero Grupo de Investigación RUECA. Correo: osiris_castilla@hotmail.com

3 Docente Institución Tecnológica Colegio Mayor de Bolívar. Coordinadora academica Institución Etnoeducativa e Inclusiva Antonia Santos. Magister en Educación. Universidad de Cartagena-SUE CARIBE. Semillero grupo de Investigacion RUECA. Correo: alejitazota@yahoo. com
} 
propósito se realizó una investigación documental y descriptiva, consultando primeramente las leyes colombianas que soportan la etnoeducacion y luego se hizo un estudio de casos de la Institución Educativa Antonia Santos en el que se evidencia el impacto de esta legislación sobre su currículo. El estudio muestra cómo desde el currículo se busca el reconocimiento de la identidad étnica y cultural en los estudiantes asumiendo su ser negro, afrodescendiente y caribeño.

Palabras clave: etnoeducación, Afrodescendientes, Política pública, Currículo, Institución Educativa.

\begin{abstract}
In recent years the Colombian legislation has wagered an inclusive, ethnic and multicultural education. These policies are the result of many years of struggle of some minorities such as African descents have historically been discriminated against, excluded and invisible. This work aims to show the Colombian ethnic education and influence public policy in the curriculum of educational institutions with a strong African identity, with reference to the educational institution Antonia Santos Cartagena, Colombia. To achieve this purpose, a documentary and descriptive research was conducted, first referring to the Colombian laws that support ethnic education and then a case study of the educational institution Antonia Santos in which the impact of this legislation on their curriculum became evident. The study shows how from the curriculum the recognition of ethnic and cultural identity in students looking assuming its black African descent and Caribbean being.
\end{abstract}


Keywords: Ethno-education, Afro-descendants, Public policy, Curriculum, Educational institution.

\section{Introducción}

Colombia es un país con una gran riqueza étnica, producto de su geografía variada y de la confluencia de culturas en distintos periodos de la historia. En la época pre-colombina coexistían en el territorio colombiano distintas familias indígenas: Chibchas, Caribes y Arawak, cada una con identidades lingüísticas y culturales propias, pero con la llegada de los españoles se produce el mestizaje y durante la colonia se impone la cultura europea con un tinte homogenizante subvalorando lo autóctono. A mediados del siglo XVI debido al comercio de esclavos entra otra cultura a nutrir el entramado étnico: la africana.

La ciudad de Cartagena fue uno de los principales puertos negreros del nuevo mundo. En palabras de Vidal (2002, p. 119):

La ciudad de Cartagena de indias, estuvo unida, desde sus comienzos hasta la abolición de la esclavitud en Colombia a la trata de negros en el Nuevo Mundo. Por su situación geográfica y por el desarrollo de su condición comercial llegó a constituirse entre los años de 1580 y 1560 en el principal depósito de esclavos en las zonas adyacentes al virreinato del Perú.

De esta forma Cartagena se convirtió en una de las ciudades colombianas con mayor número de afrodescendientes del país, en la que aún sobreviven barrios con una marcada identidad afro y en los que se conservan intactas costumbres ancestrales heredadas de esclavos libres sublevados organizados en territorios aledaños conocidos como 
Palenques, del cual el más representativo es el de San Basilio, población situada a pocos kilómetros de la ciudad.

Durante la época colonial el europeo de raza blanca estaba en la cúspide de la pirámide social e impuso su cultura utilizando la educación como principal herramienta. De esta forma la educación formal entra al país tomando como base los currículos de las escuelas de algunos países de Europa y siguiendo los preceptos de la iglesia católica, que buscando siempre la cristianización, invisibiliza la cultura del negro y cualquier otra identidad que no fuera la dominante.

A pesar de que históricamente la educación en Colombia ha estado ligada a la iglesia católica, herencia clara de los europeos, el país últimamente le ha apostado a una educación inclusiva, étnica e intercultural. Este trabajo pretende evidenciar la política pública etnoeducativa colombiana y su impacto en el currículo de las instituciones afrodescendientes, tomando como referencia la Institución Educativa Antonia Santos de la ciudad de Cartagena. 


\section{Antecedentes}

\section{Política Pública Etnoeducativa Colombiana}

Durante la época colonial la educación estuvo a cargo de la iglesia Católica. Esto es importante mencionarlo puesto que los negros no eran considerados hijos de Dios y por tanto no podían ser educados. Lago, Cassiani, \& Casseres (2015) afirman:

es la iglesia Católica la que define y designa que los negros/ as traídas de África no son hijos de Dios, por tanto no eran dignos de educación, primero era necesario hacer un proceso de conversión desde los postulados de la iglesia católica, lo que trajo consigo que por diversos se intentaba borrar todo dese su consciencia hasta su aspecto físico africano.

Es importante destacar que el concepto de escuela pública oficial se introduce de manera tardía durante el estado colonial. Jaramillo (1989) afirma:

El estado colonial solo conoció el concepto de escuela pública elemental en la segunda mitad del siglo XVIII, bajo la política ilustrada de los Borbones. En los siglos XVI y XVII la corona española impuso a los encomenderos la obligación de costear cura doctrinero para que les enseñara, a los indígenas, la doctrina cristiana, les suministrara los sacramentos y les acostumbrara a vivir... Sumisos. A pesar de esta normatividad existieron algunas escuelas de primeras letras en el siglo XVII, pero solo por la iniciativa privada, y no por una política estatal... la situación comenzó a modificarse durante la segunda mitad del siglo XVIII durante el reinado de Carlos III cuando ordenó 
dedicar parte de los bienes expropiados a los Jesuitas, para la educación. No obstante, la falta de recursos económicos aparece como el principal obstáculo para el desarrollo de este proceso .

Durante el siglo XIX la iglesia católica siguió siendo la principal institución que marcó el ritmo de la educación, tanto es así que la constitución política colombiana de 1886 ordena que la instrucción pública se haga de acuerdo a los principios religiosos. Así lo plantea Barney et al. (1989):

La historia de la educación en el último siglo muestra la íntima trabazón de las políticas educativas con los conflictos políticos e ideológicos del país: a partir de la constitución de 1886 el propio canon constitucional ordena que la instrucción pública se haga de acuerdo con los principios católicos.

Solo a partir de la Constitución Política Colombiana de 1991 se reconoce la diversidad étnica y cultural del país y se garantiza el respeto a la diferencia. En su artículo 7 se expresa "El estado reconoce y protege la diversidad étnica y cultural de la nación" y en su artículo 13 expone:

todas las personas nacen libres e iguales ante la ley, recibirán la misma protección de las autoridades y gozarán de los mismos derechos, libertades y oportunidades sin discriminación por razones de sexo, raza, origen, nacionalidad, lengua, opinión política, filosófica.

Posteriormente el artículo 68, inciso $5^{\circ}$ dice "Los integrantes de los grupos étnicos tendrán derecho a una formación que respete y desarrolle su identidad étnica y cultural". Del convenio 169 de la 
Organización Internacional del Trabajo surge la ley 21 de 1991 que en su artículo 28 establece:

Los programas y los servicios de educación destinados a los pueblos interesados deberán desarrollarse y aplicarse en cooperación con estos a fin de responder a sus necesidades particulares y deberán abarcar su historia, sus conocimientos y técnicas, sus sistemas de valores y todas las demás aspiraciones sociales, económicas y culturales.

En 1993 se aprueba la Ley 70 o Ley de Comunidades Negras. En ella se reconocen a los afrodescendientes derechos sociales, económicos, políticos, culturales y se crean las condiciones legales para el surgimiento de los programas de etnoeducación (Artículo 39). Esta ley en su artículo 32 proclama:

el estado reconoce y garantiza a las comunidades negras el derecho a un proceso educativo acorde con sus necesidades y aspiraciones etnoculturales. La autoridad competente adopta las medidas necesarias para que, en cada uno de los niveles educativos, los currículos se adapten a esta disposición.

Para darle forma a lo ordenado por la constitución de 1991 y a la ley 70 de 1993 se promulga en 1994 la ley 115 o Ley general de educación que entre sus fines persigue "Estudio y comprensión critica de la cultura nacional y de la diversidad étnica y cultural del país como fundamentos de la unidad nacional y de su identidad". Y cuyo objetivo es "fomentar el interés y el respeto por la identidad cultural de los grupos étnicos". Para dar cumplimiento al artículo 62 de esta ley surge el decreto 804 de 1995 que dice "Las comunidades afrocolombianas y raizales, en su condición de grupo étnico, les corresponde desarrollar 
procesos etnoeducativos y seleccionar educadores que sirvan a sus comunidades".

En 2010 se promulga la ley 1381 o Ley de lenguas nativas que propende por "el reconocimiento, fomento, protección uso, preservación y fortalecimiento de las lenguas de los grupos étnicos de Colombia y sobre sus derechos lingüísticos y los de sus habitantes" y que en su artículo 20 expresa:

Las autoridades educativas nacionales, departamentales, distritales y municipales y la de los pueblos y comunidades donde se hablen lenguas nativas, garantizaran que la enseñanza de estas sea obligatoria en las escuelas de dichas comunidades. La intensidad y las modalidades de enseñanza de la lengua o las lenguas nativas frente a la enseñanza del castellano, se determinarán mediante acuerdos entre las autoridades educativas del estado y las autoridades de las comunidades en el marco de procesos etnoeducativos, cuando estos estén diseñados.

Respecto a la implementación de políticas públicas etnoeducativas a nivel local, Lago, Lago y Lago (2012) exponen:

Si bien desde la ley 70 de 1993 y el decreto 2249 de 1995 crea la comisión pedagógica afroamericana en el MEN para concretar en las entidades territoriales y los líderes de las comunidades lo relacionado con la educación para los afrocolombianos, el estado colombiano inicia la apertura etnoeducativa en Cartagena De Indias, que cuenta con una gran cantidad de habitantes afrodecendietes y un gran núcleo de población marcadamente excluyente y racista solo hasta el 2 de agosto de 2004 mediante el acuerdo de 015 del consejo distrital de Cartagena De Indias adopta 
como política educativa permanente para fortalecer y reafirmar la identidad étnica y cultural, el programa de etnoeducación y diversidad cultural de la cátedra de estudios afrocolombianos en todas las instituciones educativas y establecimientos privados del distrito y todas sus localidades. Sin embargo, solo es incorporada al plan sectorial de educación 2006-2007 como componente de las competencias ciudadanas y no para todas las instituciones educativas como dispone la legislación solo para veinticinco establecimientos educativos (19 situados en los corregimientos y 6 del casco urbano) de noventa y siete (97) IED, con 258 sedes y 344 colegios privados que tiene el distrito de Cartagena. Olvidan que las minorías necesitan no solo reconocerse y respetarse, sino que los otros también las reconozcan y respeten. Hasta el momento la Secretaría de Educación Distrital (SED CARTAGENA) no ha publicado un decreto sobre etnoeducación el en distrito. La información sobre objetos, metas, procede de un documento publicado por la persona encargada de este aspecto en SED de Cartagena.

Los objetivos de la política etnoeducativa en el distrito de Cartagena son: establecer los planes curriculares de la instituciones educativas que desarrollan proyectos etnoeducativos en el distrito de Cartagena y sus localidades y promover la implementación transversal dela cátedra de estudios afrocolombianos en todos los niveles del sistema educativo, para fortalecer y reafirmar la identidad étnica y cultural, que exprese los aportes de los pueblos afrocolombianos a la construcción, formación de la nacionalidad y la identidad cartagenera. 
Las metas se orientan a la resignificación y rediseño de los PEIs y planes curriculares en 25 instituciones educativas, identificadas como etnoeducativas en el Distrito y sus localidades y a la realización de 5 encuentros sobre prácticas pedagógicas y experiencias significativas etnoeducativas y con el diseño de una Guía curricular para la implementación transversal de la cátedra de estudios afrocolombianos en las instituciones educativas del Distrito. (p. 68).

Evidentemente Colombia le ha apostado a una educación multicultural y la legislación en ese sentido es abundante. La política pública etnoeducativa ha influenciado el currículo de muchas instituciones del país y muy particularmente en Cartagena por su gran número de afrodescendientes. La Institución Educativa Antonia Santos es una de esas instituciones Cartageneras en las que se evidencia esta influencia.

\section{Resultados de la Investigación}

\section{Antonia Santos: Una Institución Etnoeducativa e Inclusiva}

La Institución Educativa Antonia Santos está ubicada en Cartagena de Indias dentro de la zona histórica y turística. Muy cerca se encuentran algunos de los monumentos más representativos de la ciudad: el castillo de San Felipe, el monumento a los Zapatos Viejos y el cerro o convento de la Popa. En las faldas de este cerro se encuentra Nariño, uno de los barrios afrodescendientes más representativos de la ciudad y del que proceden la mayoría de los estudiantes de la institución. 
Actualmente la institución se reconoce por ser etnoeducativa e inclusiva. Su proyecto etnoeducativo busca el autoreconocimiento de la identidad étnica, histórica y cultural.

... la práctica pedagógica desde una visión propia lleva a que se retomen los elementos étnicos culturales de la población palanquera ancestral para fortalecer la institución desde el preescolar y la básica primaria en las ciudades. Hablamos entonces de la música, el baile, de la historia, de la lengua materna, de la cosmovisión, de la practicas religiosas y de los principios comunitarios como la solidaridad, las familias extensas, del kuagro como elemento cohesionador de la cultura inmaterial. El autoreconocimiento se hace en doble vía; uno interno de los palenqueros y palenqueras que llegaron del pueblo a la ciudad y otro hacia la población nacida en Cartagena que tiene diversas características... Entonces surgen las preguntas ¿Cómo desarrollar en niños y niñas afro, palenqueros el aprendizaje de la lucha de los cimarrones? ¿Cómo enseñar a los estudiantes que sus ancestros fueron invisibilizados? ¿Cómo enseñar a los niños y niñas que todos somos iguales en derechos y diferentes culturalmente? (IE Antonia Santos, 2015)

La institución también atiende a población con discapacidad auditiva convirtiéndose así en un referente importante para temas de inclusión en el ámbito educativo a nivel local, regional y nacional. Hablando de inclusión Ospino (2011) afirma:

... supone grandes retos para las instituciones que deben flexibilizar sus proyectos educativos para hacerlos accesibles a personas con este tipo de limitaciones. Es cada día más frecuente encontrar instituciones educativas que deben incluir en sus aulas estudiantes sordos, ciegos, 
discapacitados, afrodescendientes y otras minorías étnicas. En el distrito de Cartagena uno de los procesos inclusivos es el de personas sordas al aula regular. Esto supone que dichas instituciones deben ajustar sus proyectos educativos para garantizar equidad en la atención a estudiantes sordos y oyentes. Como la principal barrer apara el sordo es la comunicación entonces es necesario valerse de intérpretes que traduzcan la información suministrada por los profesores en lengua de señas colombiana (p. 8).

El reconocimiento de la institución como inclusiva y etnoeducativa ha sido todo un proceso producto de su historia y de la política pública educativa colombiana. Anterior a la Ley 715 de 2001 las instituciones de educación en Colombia eran independientes y cada una tenía su propio carácter, incluso estaban clasificadas en escuelas de primaria y de secundaria. De esta forma las instituciones Alfonso Araujo, San Luis Gonzaga y Juan Salvador Gaviota, todas de primaria, tenía cada una su director y un énfasis propio: Juan Salvador Gaviota era una escuela para sordos y San Luis Gonzaga poseía una marcada identidad afrodescendiente.

Con la implementación de esta Ley se fusionan las escuelas de primaria y las de secundaria con un mismo director y un mismo proyecto educativo. Así la Concentración Educativa Antonia Santos, que era de secundaria se agrupa con las tres escuelas antes mencionadas y se forma la Institución Educativa Antonia Santos, con una sede principal y tres sedes satélite. A partir de esta evolución histórica la institución va formando su carácter pues debe permitir en su proyecto educativo la inclusión de estudiantes con discapacidad auditiva provenientes de la sede Juan Salvador Gaviota y de estudiantes afrodescendientes provenientes la sede San Luis Gonzaga. 
Desde 1999 el Proyecto Educativo Institucional de la escuela San Luis Gonzaga fue reconocido como proyecto innovador y galardonado en el 2000 como sobresaliente por el Ministerio de Educación Nacional. La Institución Educativa Antonia Santos legalmente se focaliza como una escuela etnoeducativa en 2013 a través del Decreto 0670 emanado de la Alcaldía de Cartagena.

\section{Impacto de la Política Pública Etnoeducativa en el Currículo Institucional}

\section{Resignificación del Proyecto Educativo Institucional}

Desde su focalización como institución etnoeducativa la comunidad debió reformular su objeto misional con la resignificacion de su proyecto educativo. La misión institucional se lee ahora de la siguiente manera:

Prestar servicios educativos con sentido humanístico, calidad y eficiencia para satisfacer las necesidades educativas y de formación de los educandos en el proceso de inclusión y etnoeducacion, proyecto bandera institucional, hacia la búsqueda continua y permanente de la excelencia educativa a fin de lograr óptimos desempeños en nuestros estudiantes en los diferentes contextos familiares, laborales y socioculturales. (IE Antonia Santos, 2015)

La visión institucional también se reformula:

... la Institución Educativa Antonia Santos en su condición de líder en el proceso de inclusión y etnoeducacion se consolidará en la ciudad como una comunidad multicultural de alto nivel académico, tecnológico, 
deportivo e investigativo y en valores, constituyéndose así en una institución fortalecida en el logro de la formación y educación de futuros hombre y mujeres colombianos.

De esta reformulación de la misión y visión institucional parte un proceso muy nutrido de experiencias significativas en la práctica educativa en la búsqueda siempre de la identidad cultural y de la inclusión.

\section{Nuevo Enfoque del Plan de Estudios}

El impacto de la política etnoeducativa en el plan de estudios se evidencia en la creación de la asignatura de etnoeducación y su introducción progresiva, primero en básica primaria y luego en secundaria. Este cambio implicó reestructurar el área de ciencias sociales, asignando una hora semanal al estudio de esta asignatura. Dentro de los propósitos del área se persigue:

- La enseñanza de la historia ancestral a partir de la reconstrucción de los aportes de los afrodescendientes en la lucha por la libertad, la autonomía, la independencia y la resistencia en general.

- El fortalecimiento de la identidad étnica y cultural, logrando que los estudiantes identifiquen y asuman su ser negro, afro, palenquero, raizal, mestizo o indígena.

- Sistematizar la experiencia etnoeducativa en diversos medios de comunicación, desde el uso de las nuevas tecnologías hasta la elaboración de un periódico con la participación de toda la comunidad educativa, en donde el escribir y el leer sean eje central de la cotidianidad académica. 
- El uso de pedagogías propias entendidas como los diferentes métodos y estrategias que de generación en generación han permitido la supervivencia y transmisión del conocimiento ancestral.

\section{Escuela de Lengua Criolla Palenquera}

La lengua criolla palenquera es la lengua hablada en San Basilio de Palenque, población situada a pocos kilómetros de la ciudad y de la cual son descendientes la mayoría de los estudiantes de la institución. Esta lengua tiene una fuerte influencia de lenguas romances como el portugués y el castellano y de lenguas bantúes. ${ }^{4}$ Con la escuela de lengua criolla se busca que los estudiantes reconozcan y aprendan la lengua de sus antepasados y se apropien de esta como patrimonio intangible protegido por la ley 1381 o Ley de lenguas nativas que propende por "el reconocimiento, fomento, protección uso, preservación y fortalecimiento de las lenguas de los grupos étnicos de Colombia y sobre sus derechos lingüísticos y los de sus habitantes".

\section{Escuela de Danza y Música Joe Arroyo}

La cadencia en el baile es una de las herencias africanas mayormente marcadas en la población cartagenera. La fusión de los ritmos africanos ancestrales interpretados en tambores y llamadores con otros ritmos caribeños ha originado géneros musicales como la salsa, cuyo máximo exponente a nivel regional es el cartagenero Joe Arroyo, ya fallecido, pero nacido y criado en el popular barrio Nariño, de donde proceden la mayoría de los estudiantes de la institución. En homenaje a este gran artista se ha creado la escuela de danza y música Joe Arroyo

4 Lenguas habladas por individuos pertenecientes a grupos étnicos africanos situados desde Camerún hasta Somalia. 
que busca fortalecer la identidad a través de los ritmos africanos. Los estudiantes ingresan interesados en el baile desde muy temprana edad y se les enseñan ritmos como el mapalé, la cumbia, la salsa, el merengue, el bullerengue, entre otros.

\section{Dotación a la Biblioteca de Material Etnoeducativo}

Al implementar la cátedra de etnoeducación se hace necesario dotar la biblioteca de una colección especial de títulos sobre el área. En esta sección se recopilaron algunos libros de escritores afro locales, regionales y nacionales. La colección tiene ejemplares de arte afro, poesía negra, lengua criolla palenquera, historias en caricaturas, investigaciones sociológicas y antropológicas, cartillas seriadas, publicaciones del ministerio de educación, entre otras.

\section{Periódico Escolar Etnoeducativo}

El periódico escolar etnoeducativo es un órgano de difusión de noticias creado con la participación de toda la comunidad educativa con la finalidad de que el escribir y el leer sean eje central de la cotidianidad académica. Es una herramienta muy útil tanto para el área de castellano como para el componente transversal de otras áreas. Para el periódico escriben estudiantes, profesores, directivos docentes y otros miembros de la comunidad.

\section{Practicas Pedagógicas}

Debido al carácter etnoeducativo de la institución y de acuerdo a los lineamientos curriculares antes mencionados, las diferentes áreas 
manejan proyectos que integran saberes universales y saberes ancestrales de las comunidades afrodescendientes.

Desde el área de matemáticas por ejemplo se han realizado trabajos comparativos de los sistemas de medición actuales con los sistemas de medición ancestrales, así como también se estudia la geometría de los peinados típicos afrocaribeños.

En las ciencias naturales se estudian y cultivan algunos tipos de semillas de plantas que tradicionalmente han sido usadas por estas comunidades para su alimentación y como medicinas. En las ciencias sociales se estudia el tema afrocaribeño desde diferentes perspectivas: histórica, geográfica, estética y folclórica. La parte folclórica es muy rica en expresiones culturales como los bailes, la gastronomía, los trajes típicos, la estética del negro, los peinados.

\section{Escuela de danza y música Joe Arroyo}

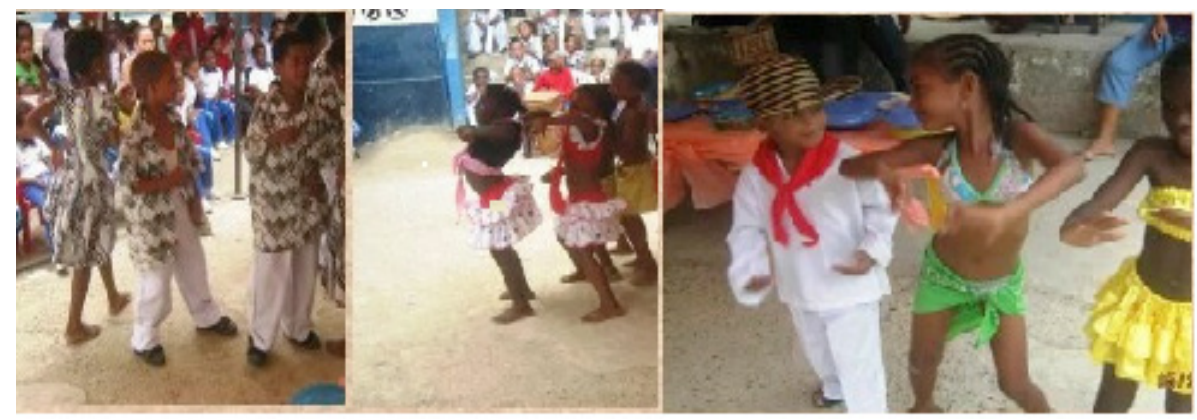

Fuente: fotografía archivo personal de profesora Mónica Reyes. 
Geometría de los peinados típicos afrocaribeños

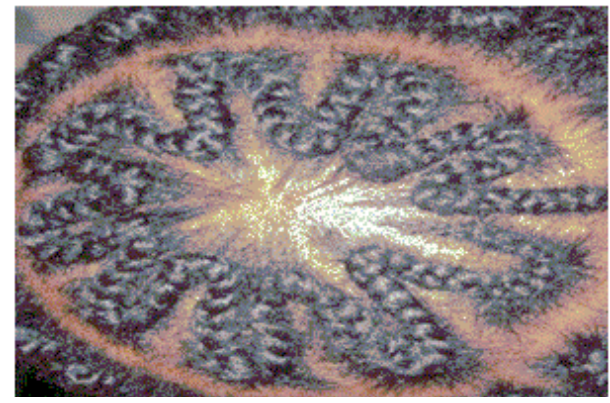

Fuente: Fotografía archivo personal profesora Maricel Jiménez.

\section{Cultivos de semillas ancestrales}

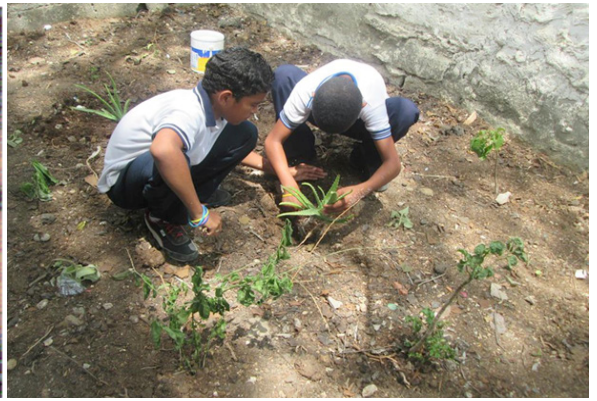

Fuente: Fotografía archivo personal profesora Mónica Reyes.

Fuente: fotografía archivo personal profesor Oscar Ospino

\section{Impacto de la Política Pública Etnoeducativa en la Construcción de Identidad Étnica}

\section{Construcción de identidad étnica en la escuela}

A través de las acciones mencionadas anteriormente tales como la resignificación del proyecto educativo, la inclusión de la asignatura de etnoeducación en el plan de estudios y el enriquecimiento de la práctica pedagógica a través de la gestión cultural se ha logrado la construcción de identidad étnica en la escuela. Obviamente esto se ha potenciado desde la focalización de la institución como etnoeducactiva, tanto así que a nivel local es un referente importante en esta área llegando a ser sede y anfitriona de numerosos eventos afrocolombianos en la ciudad tales como encuentros, talleres, y simposios. Internacionalmente la institución ha participado en numerosos congresos y eventos académicos con intercambio de experiencias significativas en el área de etnoeducación. Estos eventos académicos se han realizado en 
importantes universidades como la Universidad Autónoma de Tlaxcala (México) y la Universidad The west Indies (Jamaica).

\section{Construcción de identidad étnica en la comunidad}

Las acciones de construcción de identidad que se han dado desde la escuela han impactado la comunidad entera, fortaleciendo las tradiciones culturales familiares ancestrales, así como también la vida de barrio y de vecindad. La participación de las familias se ha hecho evidente en su integración con las actividades que se planifican desde la escuela creando aprendizajes en doble vía: en la escuela se imparte desde el currículo los saberes ancestrales y desde la familia se contextualizan con las prácticas comunitarias. Un claro ejemplo de esto es el aprendizaje de la lengua criolla palenquera: desde la escuela se estudian los componentes semánticos, lingüísticos y sintácticos de lengua, pero desde la familia y desde el vecindario se refuerza con la práctica y la escucha de los adultos mayores que la mantienen viva.

\section{Dificultades del proceso}

El proceso de construcción de identidad no ha estado exento de dificultades. Uno de los mayores obstáculos es la subvaloración de lo autóctono potenciado por los medios de comunicación masivos que imponen culturas extranjeras como referentes de vida y de costumbres. Los jóvenes entonces se sienten atraídos por ritmos, lenguas y costumbres extranjeras que les hacen creer que lo autóctono es de menor valor. Afortunadamente ha prevalecido la identidad y las políticas públicas gubernamentales apuntan al rescate de las tradiciones culturales reivindicando los saberes ancestrales. 


\section{Conclusiones}

La política pública educativa colombiana en los últimos años ha reivindicado el derecho a una educación inclusiva, étnica y multicultural de algunas minorías como los afrodescendientes. La legislación en ese sentido ha sido bastante amplia y busca proteger su legado cultural, sapiencial y lingüístico.

Las instituciones educativas que atienden población afrodescendiente han tenido que adaptar su currículo a los retos que implica el reconocimiento de la identidad, reformulando su filosofía, objeto misional y proyecto educativo.

La política pública etnoeducativa colombiana ha influenciado los planes de estudio de las instituciones que atienden población afrodescendiente. Esto se evidencia en la introducción de nuevas asignaturas que buscan el fortalecimiento de la identidad étnica, cultural y lingüística.

La Institución Educativa Antonia Santos evidencia el impacto de estas políticas en su currículo, sustentado en un objeto misional orientado al reconocimiento de su identidad afrodescendiente y en las prácticas pedagógicas que cohesionan los saberes universales con los saberes ancestrales. 


\section{Referencias}

Barney Cabrera, E., Camacho Guizado, E., Colmenares, G., Corradine Angulo, A., Cristina Zonza, M., Diaz Diaz, F., .. Tirado Mejía, A. (1989).

Nueva Historia de Colombia. Bogotá, D.C. Colombia: Editorial Pinter Colombia Ltda.

Congreso de Colombia. Ley 21 de 1991 (1991). República de Colombia: Diario

Oficial 39.720 de marzo 6 de 1991. Recuperado a partir de http://www. alcaldiabogota.gov.co/sisjur/normas/Normal.jsp?i=37032

Congreso de Colombia. Ley 70 (1993). República de Colombia: Diario Oficial

No. 41.013. Recuperado a partir de http://www.alcaldiabogota.gov.co/ sisjur/normas/Normal.jsp?i=7388

Congreso de Colombia. Ley 115 de 1994 (1994). República de Colombia:

Diario Oficial No. 41.214 de 8 de febrero de 1994. Recuperado a partir

de https://www.mineducacion.gov.co/1621/articles-85906_archivo_ pdf.pdf

Congreso de Colombia. Ley 715 de 2001 (2001). República de Colombia:

Diario Oficial No 44.654, de 21 de diciembre de 2001. Recuperado

a partir de http://observatorioetnicocecoin.org.co/cecoin/files/ Ley_715_2001.pdf

Congreso de Colombia. Ley 1381 de 2010 (2010). República de Colombia:

Diario Oficial No. 47.603, de enero 25 de 2010. Recuperado a partir

de http://www.mincultura.gov.co/areas/poblaciones/publicaciones/

Documents/Ley de Lenguas 2013.pdf

Constitución Política de Colombia. Artículo 13 (1991). República de Colombia.

Constitución Política de Colombia. Artículo 68 (1991). República de Colombia. Constitución Política de Colombia. Artículo 7 (1991). República de Colombia. IE Antonia Santos. (2015). Plan de area de etnoeducacion: conocimiento propio, intangible e inmaterial de los grupos etnicos. Cartagena, Colombia.

Jaramillo Uribe, J. (1989). El proceso de la educación en el virreinato. En Nueva Historia de Colombia. Bogotá, D.C. Colombia: Planeta.

Lago, A., Lago, C., \& Lago, D. (2012). Educación Para Ciudadanos Del Mundo Con Identidad Afrodescendiente: Caso Institución Educativa Antonia Santos, Cartagena De Indias, Colombia. Revista Historia de la Educación Latinoamericana, 14(18), 53-74. Recuperado a partir de http://www.redalyc.org/articulo.oa?id=86925890003

Lago de Zota, A., Cassiani Herrera, T., \& Casseres Gomez, D. (2015). Enfoques curriculares etnoeducativos e Inclusion, una experiencia significativa. 
En Congreso Internacional de Educacion: Currículum. Tlaxcala: Universidad Autónoma de Tlaxcala. Recuperado a partir de https:// posgradoeducacionuatx.org/pdf2015/A036.pdf

Ospino Guerrero, O. J. (2011). Diseño de un software educativo de orientación vocacional con traducción multimedial en lengua de señas colombiana. Universidad Autónoma de Bucaramanga, UNAB. Universitat Oberta de Catalunya, UOC, Cartagena de Indias. Recuperado a partir de http:// sibu.unab.edu.co/cgi-bin/koha/opac-detail.pl?biblionumber=155483

Presidencia de la República. Decreto 804 de 1995 (1995). República de Colombia: Diario Oficial No. 41853 de mayo 18 de 1995. Recuperado a partir de http://www.alcaldiabogota.gov.co/sisjur/normas/Normal. jsp?i=1377

Vidal, A. (2002). Cartagena de Indias y la región Histórica del Caribe, 15801640. Sevilla: Secretariado de Publicaciones de la Universidad de Sevilla. 